\title{
ON NONCLASSICAL IMPULSIVE ORDINARY DIFFERENTIAL EQUATIONS WITH NONLOCAL CONDITIONS
}

\author{
S. A. BISHOP*, M. C. AGARANA AND J. G. OGHONYON \\ Department of Mathematics, Covenant University, Ota, Ogun State, Nigeria \\ ${ }^{*}$ Corresponding author: sheila.bishop@covenantuniversity.edu.ng
}

\begin{abstract}
Results on mild solutions of nonclassical differential equations with impulsive and nonlocal conditions are extended to a case when the nonlocal conditions are necessarily non Lipschitz and non compact.
\end{abstract}

\section{INTRODUCTION}

We study the following quantum stochastic differential equation (QSDE) with impulsive nonlocal conditions introduced in [1];

$$
\begin{aligned}
d z(t)= & A(t) z(t)+E\left(t,(z(t)) d \wedge_{\pi}(t)+F(t, z(t)) d A_{f}(t)\right. \\
& +G(t, z(t)) d A_{g}^{+}(t)+H(t, z(t)) d t, \\
& \text { almost all } t \in I, t \neq t_{k}, k=1, \ldots, m \\
& \Delta z\left(t_{k}\right)=J_{k}\left(z\left(t_{k}^{-}\right)\right), k=1, \ldots, m \\
& z\left(t_{0}\right)=z_{0}+g(z), \quad t \in[0, T]
\end{aligned}
$$

where

(i) A is a family of semigroup defined in [1]

Received 2018-07-04; accepted 2018-09-12; published 2019-01-04.

2010 Mathematics Subject Classification. 35A24.

Key words and phrases. nonclassical ordinary differential equations (NODEs); non-compact; nonlocal conditions; impulse effect; non Lipschitz; Stochastic processes.

(C)2019 Authors retain the copyrights of their papers, and all open access articles are distributed under the terms of the Creative Commons Attribution License. 
(ii) E, F, G, H are stochastic processes.

(iii) $J_{k} \in C(\tilde{\mathcal{B}}, \tilde{\mathcal{B}}), k=1,2, \ldots, m$ and $\Delta z\left(t_{k}\right)$ is the difference between $z\left(t^{+k}\right)$ and $z\left(t_{k}^{-}\right)$.

(v) $g: \tilde{\mathcal{B}} \rightarrow P C(I, \operatorname{ses} q(\mathbb{D} \underline{\otimes} \mathbb{E}))$ is a nonlocal condition that is not Lipschitz and not compact.

(vi) $z \in \tilde{\mathcal{B}}$ is a stochastic process and $\eta, \xi \in \mathbb{D} \underline{\otimes \mathbb{E}}$ is arbitrary.

Problems with nonlocal conditions have been an area of interest, mostly because of the advantage they have over initial value problems. Existence of solution of nonlocal problems for different types of differential equations were extensively discussed in the literature by using various methods (See [1, 2, 3-14] and the references therein). The motivation for this study, is that nonlocal problems occur naturally when modeling physical problems. In [2], impulsive quantum stochastic differential equations (IQSDE) with initial value conditions were studied. The multivalued maps are lower respectively upper semicontinuous. In [1], existence results for Eq.(1) with nonlocal conditions that are completely continuous were established. We showed that the function $g$ which constitute the nonlocal condition is compact and Lipschitz continuous. Several interesting results on nonlocal impulsive differential equations satisfying some Lipschitz and compactness conditions have been established in [6-9]. In this study, existence of solution of Eq. (1) is established with nonlocal conditions that are not necessarily Lipschitz and compact. We adopt the most suitable fixed point method to establish this result. Impulsive QSDEs have found applications in quantum continuous measurements, especially when the mean number of photons up to time $t_{i}$ is momentary giving rise to impulses on the counting stochastic processes associated with the observables $x\left(t_{i}\right)$. See $[1,2]$ and the references therein.

\section{Preliminaries}

The definitions of the following spaces $L_{\text {loc }}^{2}(\tilde{\mathcal{B}})_{m v s}, \tilde{\mathcal{B}}, P C(I, \tilde{\mathcal{B}}), P C^{\prime}(I, \tilde{\mathcal{B}}), P C(I, \operatorname{ses} q(\mathbb{D} \underline{\otimes}))$ and $P C^{\prime}(I, \operatorname{ses} q(\mathbb{D} \underline{\otimes}))$ are adopted from $[1,2]$. The spaces $\tilde{\mathcal{B}}$ and $P C(I, \operatorname{ses} q(\mathbb{D} \underline{\otimes}))$ denote the locally convex and Banach spaces respectively. The Hausdorff distance, $\rho(A, B)$ is defined as:

$$
\rho(A, B)=\max (\delta(A, B), \delta(B, A)), A, B \in \operatorname{clos}(\mathbb{C})
$$

and

$$
d(z, B)=\operatorname{Inf}_{y \in B}|z-y|, \quad \delta(A, B)=\operatorname{Sup}_{z \in A} d(z, B)
$$

where $x \in \mathbb{C}$ is as defined in [1] and $\rho$ is a metric.

Definition 1. A stochastic process $z \in P C(I, \tilde{\mathcal{A}})$ is called a solution of Eq. (1) if it satisfies the integral 
equation

$$
\begin{aligned}
z(t)= & S(t)\left[z_{0}+g(z)\right] \\
& +\int_{0}^{t} S(t-s)\left(E \left(s,(z(s)) d \Lambda_{\pi}(s)+F(s, z(s)) d A_{f}(s)\right.\right. \\
& \left.+G(s, z(s)) d A_{g}^{+}(s)+H(s, z(s)) d s\right)+\sum_{0<t_{k}<t} S\left(t-t_{k}\right) J_{k}\left(z\left(t_{k}\right)\right)
\end{aligned}
$$

for $t \in[0, T]$. To establish a mild solution with non Lipschitz, non compact and nonlocal condition, we must assume that $\mathrm{z}(\mathrm{t})$ near zero does not affect the nonlocal condition $\mathrm{g}(\mathrm{z})$. We use the equivalent form of Eq. (1) given by,

$$
\frac{d}{d t}\langle\eta, z(t) \xi\rangle=P(t, z(t))(\eta, \xi)
$$

Remark. Equation (3) is also known as nonclassical ordinary differential equation. See [1] and the references therein. Next, we state the following assumptions:

$\left(H_{1}\right)$ The map $P$ in Eq. (3) is continuous and well defined in [1]. Hence, there exists a function $K_{\eta \xi}^{P}$ : $[0, T] \rightarrow \mathbb{R}_{+}$so that

$$
\|P(t, y)-P(t, z)\|_{\eta \xi} \leq K_{\eta \xi}^{P}(t)\|y-z\|_{\eta \xi}
$$

$t \in[0, T], y, z \in \tilde{\mathcal{B}}$.

$\left(H_{2}\right) \quad J_{k} \in C(\tilde{\mathcal{B}}, \tilde{\mathcal{B}})$ and $T($.$) are compact operators$

$\left(H_{3}\right) \quad$ For each $z_{0} \in \tilde{\mathcal{B}}$, we have constants $h_{\eta \xi}>0$ and $M>0$ so that

$$
\|S(t)\|_{\eta \xi} \leq M, t \geq 0
$$

and

$$
M\left(\left\|z_{0}\right\|_{\eta \xi}+\sup _{\varphi \in H_{h}}\|g(\varphi)\|_{\eta \xi}+K_{\eta \xi}^{P}(t) \sup _{s, t \in[0, T]}\|P(s, \varphi(s))\|_{\eta \xi}+\sup _{\varphi \in Z_{h}} \sum_{k=1}^{m}\left\|J_{k}\left(\varphi\left(t_{k}\right)\right)\right\|_{\eta \xi}\right) \leq h_{\eta \xi}
$$

where

$$
H_{h}:=\left\{\varphi \in P C([0, T], \tilde{\mathcal{A}}):\|(\varphi(t))\|_{\eta \xi} \leq h_{\eta \xi}, t \in[0, T]\right\}
$$

$\left(H_{4}\right) \quad g: P C([0, T], \tilde{\mathcal{A}}) \rightarrow \tilde{\mathcal{A}}$ is continuous and constitute the nonlocal condition. Also $g: H_{h} \rightarrow b d$ Let $\delta$ depend on $h_{\eta \xi} \in\left(0, t_{1}\right)$ and $g(\varphi)=g(\phi), \varphi, \phi \in H_{h}$ where $\varphi(s)=\phi(s), s \in[\delta, T]$ and bd denote a bounded set. 


\section{Main Result}

Theorem 1. Let conditions $\left(H_{1}\right)-\left(H_{4}\right)$ hold. Then for $z_{0} \in \tilde{\mathcal{B}}$, problem (1) has at least a solution. Proof. Let $\delta \in\left(0, t_{1}\right)$, define $H(\delta)$ and $H_{h}(\delta)$ as ;

$$
H(\delta):=P C([\delta, T], \tilde{\mathcal{B}}) \text { for functions in } P C([0, T], \tilde{\mathcal{B}}) \text { on }[\delta, T]
$$

and

$$
H_{h}(\delta):=\left\{\varphi \in H(\delta):\|\varphi(t)\|_{\eta \xi} \leq h_{\eta \xi}, t \in[\delta, T]\right\} .
$$

Let $z \in H_{h}(\delta)$ be fixed. Then define a map $\Gamma_{z}$ on $H_{h}$ by

$$
\begin{aligned}
\Gamma_{z}(\varphi)(t)(\eta, \xi)= & \left\langle\eta,\left[z_{0}+g(\tilde{z})\right] \xi\right\rangle \\
& +\int_{0}^{t} S(t-s) P(s,(\varphi(s))(\eta, \xi) d s \\
& +\sum_{0<t_{k}<t} S\left(t-t_{k}\right) J_{k}\left(u\left(t_{k}\right)\right), t \in[0, T], \tilde{z} \in H_{h}(\delta)
\end{aligned}
$$

where

$$
\tilde{z}(t)=z(t), \text { if } t \in[\delta, T]
$$

and

$$
\tilde{z}(t)=z(\delta), \text { if } t \in[0, \delta]
$$

This shows that $\Gamma_{z}$ is a continuous mapping from $H_{h}$ to $H_{h}$. Also, we have

$$
\begin{aligned}
\left\|\Gamma_{z}^{n}(\varphi)(t)-\Gamma_{z}^{n}(\phi)(t)\right\|_{\eta \xi} & \\
& =\left|\int_{0}^{t} S(t-s)(P(s, \varphi(s))(\eta, \xi)-P(s, \phi(s))(\eta, \xi) d s)\right| \\
& \leq M \int_{0}^{t}\|P(s, \varphi(s))-P(s, \phi(s))\|_{\eta \xi} d s \\
& \leq M \int_{0}^{t} K_{\eta \xi}^{P}(s)\|\varphi(s)-\phi(s)\|_{\eta \xi} d s
\end{aligned}
$$

Since the map $s \rightarrow \sup _{s \in[0, T]}\|\varphi(s)-\phi(s)\|_{\eta \xi}$ is continuous, we let

$$
R_{\eta \xi}=\sup _{s \in[0, t]}\|\varphi(s)-\phi(s)\|_{\eta \xi}
$$

and

$$
N_{\eta \xi}(t)=\int_{0}^{t} K_{\eta \xi}^{P}(s) d s
$$

Then from (3), we get

$$
\left\|\Gamma_{z}^{n}(\varphi)(t)-\Gamma_{z}^{n}(\phi)(t)\right\|_{\eta \xi} \leq \frac{R_{\eta \xi}\left(M N_{\eta \xi}(t)\right)^{n}}{n !}, n=1,2, \ldots
$$


This can also be proved by induction, see [7] in [1].

So we get

$$
\begin{aligned}
\left\|\Gamma_{z}^{m}(\varphi)(t)-\Gamma_{z}^{m}(\phi)(t)\right\|_{\eta \xi}= & \left\|\sum_{m=k+1}^{n}\left(\Gamma_{z}^{m}(\varphi)(t)-\Gamma_{z}^{m}(\phi)(t)\right)\right\|_{\eta \xi} \\
& \leq \sum_{m=k+1}^{n}\left\|\left(\Gamma_{z}^{m}(\varphi)(t)-\Gamma_{z}^{m}(\phi)(t)\right)\right\|_{\eta \xi} \\
& \leq \sum_{m=k+1}^{n} \frac{R_{\eta \xi}\left(M N_{\eta \xi}(T)\right)^{m}}{m !}
\end{aligned}
$$

where $t \in[0, T], \varphi, \phi \in H_{h}, m=1,2, \ldots$

Now by considering large $\mathrm{m}$, we see that $\Gamma_{z}^{m}$ is a contraction operator on $H_{h}$ and hence $\Gamma_{z}$ has a unique fixed point given by

$$
\begin{aligned}
\varphi_{z}(t)(\eta, \xi)= & \left\langle\eta,\left[z_{0}+g(\tilde{z})\right] \xi\right\rangle \\
& +\int_{0}^{t} S(t-s) P\left(s, \varphi_{z}(s)\right)(\eta, \xi) d s \\
& +\sum_{0<t_{k}<t} S\left(t-t_{k}\right) J_{k}\left(z\left(t_{k}\right)\right) \in H_{h}, t \in[0, T]
\end{aligned}
$$

From the above, define a map $\Upsilon: H_{h}(\delta) \rightarrow H_{h}(\delta)$ by

$$
\begin{aligned}
\Upsilon(z)(t)(\eta, \xi)=\varphi_{z}(t)(\eta, \xi)= & \left\langle\eta,\left[z_{0}+g(\tilde{z})\right] \xi\right\rangle \\
& +\int_{0}^{t} S(t-s) P\left(s, \varphi_{z}(s)\right)(\eta, \xi) d s \\
& \leq \sum_{0<t_{k}<t} S\left(t-t_{k}\right) J_{k}\left(z\left(t_{k}\right)\right), t \in[\delta, T]
\end{aligned}
$$

The next following steps show that the map $\Upsilon$ has a fixed point:

By applying Lebesgue dominated convergence theorem and Arzela-Ascoli theorem we show that; (1) the operator $\Upsilon$ is continuous;

(2) It maps bd sets into bd sets in $P C(I, \operatorname{sesq}(\mathbb{D} \underline{\otimes} \mathbb{E}))$;

(3) $\Upsilon$ maps bd sets into equicontinuous sets in $P C(I, \operatorname{ses} q(\mathbb{D} \underline{\otimes \mathbb{E}}))$ and by applying the Schauder-Tychonov's fixed point theorem we get a $z^{*} \in H_{h}(\delta)$, which is a fixed point.

By replacing $\Upsilon(z)$ with $z, g(\tilde{z})$ with $g\left(\tilde{z}^{*}\right)$ in (5), we get

$$
\begin{aligned}
z(t)(\eta, \xi)= & \left\langle\eta,\left[z_{0}+g\left(\tilde{z}^{*}\right)\right] \xi\right\rangle \\
& +\int_{0}^{t} S(t-s) P(s, z(s))(\eta, \xi) d s \\
& \leq \sum_{0<t_{k}<t} S\left(t-t_{k}\right) J_{k}\left(z^{*}\left(t_{k}\right)\right), t \in[0, T]
\end{aligned}
$$


But

$$
g\left(\tilde{z}^{*}\right)=g(\tilde{z}), \tilde{z}^{*}\left(t_{k}\right)=\tilde{z}\left(t_{k}\right)
$$

and by the definition of the map $\Upsilon$,

$$
\tilde{z}^{*}(t)=\Upsilon\left(\tilde{z}^{*}\right)(t)=\varphi_{\tilde{z}^{*}}(t)=\tilde{z}(t)
$$

So that by (6), we conclude that $z(t)$ is the required solution of Eq. (1) which is the desired result.

\section{Conclusion}

Using the equivalent form of QSDE (1) given by Eq. (3) and having satisfied the conditions of the appropriate fixed point theorem, we conclude that a fixed point exists and it is a solution of the problem.

\section{Conflict of Interest}

The authors declare that there is no conflict of interests.

\section{REFERENCES}

[1] S. A. Bishop, E. O. Ayoola, J. G. Oghonyon, Existence of mild solution of impulsive quantum stochastic differential equation with nonlocal conditions. Anal. Math. Phys. 7 (3) (2017), 255-265.

[2] S. A. Bishop and P. E. Oguntunde, Existence of Solutions of Impulsive Quantum Stochastic Differential Inclusion, J. Eng. Appl. Sci. 10 (7) (2015), 181-185.

[3] T. Caraballo, M. A. Hammami, L. Mchiri, Practical exponential stability of impulsive stochastic functional differential equations. Syst. Control Lett. 109 (2017), 43-48.

[4] T. Cardinali, P. Rubbioni, Nonlinear Anal., Theory Methods Appl. 75 (2) (2012), 871-879.

[5] M. A. Dialloa, K. Ezzinbib, A. Sene, Impulsive integro-differential equations with nonlocal conditions in Banach spaces. Trans. A. Razmadze Math. Inst. 171 (2017), 304-315.

[6] Q.Dong, G. Li, Existence of solutions for semilinear differential equations with nonlocal conditions in Banach spaces. Electron J. Qual. Theory Diff. Equ. 47 (2009), Art. ID 47.

[7] Z.B. Fan, G. Li, Existence results for semilinear differential equations with nonlocal and impulsive conditions. J. Funct. Anal. 258 (2010), 1709-1727.

[8] G. Li, Existence results for semilinear differential equations with nonlocal conditions and impulsive conditions. J. Funct. Anal. 258(2010), 1709-1727.

[9] L. Zhu , Q. Dong and G. Li, Impulsive differential equations with nonlocal conditions in general Banach spaces. Advances in Diff. Equ. 2012 (2012), Art. ID 10.

[10] A. G. Okeke, S A. Bishop,S. H. Khan Iterative Approximation of Fixed Point of Multivalued $\rho$-Quasi-Nonexpansive Mappings in Modular Function Spaces with Applications, J. Funct. Spaces 2018 (2018), Art. ID 1785702.

[11] O. Bolojan-Nica, G. Infante, R. Precupa, Existence results for systems with coupled nonlocal initial conditions. Nonlinear Anal., Theory Methods Appl. 94 (2014), 231-242

[12] S. Ji and G. Li, A unified approach to nonlocal impulsive differential equations with the measure of noncompactness. Adv. Diff. Equ. 2012 (2012), Art. ID 182. 
[13] X.M. Xue, Semilinear nonlocal problems without the assumptions of compactness in Banach spaces. Anal. Appl. 8 (2010), 211-225.

[14] X.M, Xue, Nonlocal nonlinear differential equations with a measure of noncompactness in Banach spaces.Nonlinear Anal., Theory Methods Appl. 70 (2009), 2593-2601. 